\title{
Utilising Equipment Matrices for Information Technology in Primary School Education Policy
}

\author{
Anita Greenhill \\ University of Salford, Greater \\ Manchester, England
}

A.G.Greenhill@salford.ac.uk

\author{
Gordon Fletcher \\ Griffith University, Brisbane, \\ Australia
}

G.Fletcher@griffith.edu.au

\section{Abstract}

This paper is a reflective discussion on the use of equipment matrices to determine infrastructure requirements in an education context. This position was originally presented within the wider framework of a governmentfunded research project to initiate national policies for implementing IT within primary schools. Equipment matrices were seen by the policymakers funding this reasearch as an appropriate method for representing the needs of a school.

Equipment matrices represent a systematised and regularised understanding of the relationships between social practices and technological tools (Curriculum Materials Information Services, 1997). The users of these tools are enmeshed within the matrix through a complex combination of meanings and interaction. However, the correlation of variables within a two-dimensional matrix produces a 'simple' representation of the available information that is heavily abbreviated. Lost among this condensation are the needs and presence of the user, either individually or collectively. The 'snapshot' of information that matrices present is, however, refined by the direct inclusion of volatile information such as contemporary equipment and sofware specifications.

In this paper we argue that the range of factors beyond technical specifications that influence the use and understanding of information technology are necessary elements within any consideration of IT infrastructure requirements. These, however, can only be simultaneously included in the equipment matrix representation with more expansive incorporation of multiple parameters. Simplification, we advocate, should not be the aim of the methods that determine educational infrastructure requirements but rather, in its place, is the need for sensitivity to the learners and their needs.

Keywords: Education Policy, Infrastructure, Student-Focussed Education

\section{Introduction}

This paper reflects upon the use of equipment matrices by government and other education policy-makers to determine the infrastructure requirements of schools and classrooms. This discussion was originally presented

Material published as part of these proceedings, either on-line or in print, is copyrighted by Informing Science. Permission to make digital or paper copy of part or all of these works for personal or classroom use is granted without fee provided that the copies are not made or distributed for profit or commercial advantage AND that copies 1) bear this notice in full and 2) give the full citation on the first page. It is permissible to abstract these works so long as credit is given. To copy in all other cases or to republish or to post on a server or to redistribute to lists requires specific permission from the publisher at Publisher@InformingScience.org within the wider framework of a government-funded research project to initiate national policies for incorporating IT facilities within primary schools in Australia. The brief for this academic research was broad. The researchers involved felt that this reflected an open attitude by the government commissioning the work to be receptive to new angles and positions. In light of this assumed receptiveness it was seen as cu- 
rious that the project was required to include a discussion of equipment matrices. The insistence for a discussion of this type appeared somewhat incongruous with other aspects of the research project that specifically acknowledged the significance of students, their teachers, their families and their principals in relation to the use of IT in the education environment. The educational philisophy of this research specifically sought to enhance the facilitation of lifelong learning capabilities and generic skills. The research advocated the need for acknowledgement and understanding of cultural differences, cultural capital, professional development requirements and computer usage outside the formal education setting as integral points for consideration in the policy making process. The requirement to include what is primarily a method for representing physical technological requirements in the classroom was apparently at odds with discussions of the role and actions of stakeholders in the IT education debate. Equipment matrices were, and still remain, one of the few ways in which policy-makers represent, understand and associate the technology requirements of stakeholders and their schools.

The equipment matrix takes a variety of forms in policy documents. Some are presented as graphical matrices while others are incorporated in the body of the policy. With regular occurrence these matrices focus upon the equipment in isolation from the theoretical foundations, practical considerations and cultural conditions acknowledged and presented by these policies or the schools that are the subject of the discussion.

The Kansas State Department of Education offers guidance for schools in its Local Technology Planning Guide (2000). This includes the suggestion to, "Define the minimum capabilities to meet the student outcomes (e.g., do not list a computer with a $1000 \mathrm{MB}$ hard drive and $16 \mathrm{MB}$ RAM when a $500 \mathrm{MB}$ hard drive and * MB RAM is sufficient). The same situation applies to software needs." Graphical matrices convey even more powerful representations of the technology situation in the classroom that can over-emphasize its importance in the context of the policy. The Education Technology: A Guide for School Districts (2001) developed and adopted by the California Department of Education offers a matrix within its planning documentation examples (Table 1). This matrix reduces the focus of concern to the number of physical machines in a school with or without an Internet connection. While this matrix is part of a larger Technology Planning Toolkit the graphical nature of the representation and the seeming cultural neutrality of this matrix offers what appears to be a simplistic solution to the complexities of information technology in schools.

The danger of equipment matrices, and the concern of this paper, is in their application to the learning environment. It is clear from the authors' recent personal experiences that despite equipment matrices representing only part of the complexity of the IT in schools debate their attention to the tangible aspects of technology, the vendor-supplied hardware and software, subtlely and silently becomes too easily the entire solution to this contemporary social problem. Schools are outfitted with hardware and software to a specification determined by a single education authority with little or no regard to regional variations, skill levels amongst students and teachers, ongoing training or support. In some extreme cases, schools are left with incorrectly installed or dysfunctional systems. A debate that is currently receiving attention in the United Kingdom (which was not the location of the original research) is the argument that high bandwidth Internet access for schools, and more generally, should be treated as a utility in the same context as electricity or water. There are already indications that the central government may accept this argument by pursuing policies that support this claim (Arnott 2002, 4). However, this remains a policy in potentia without a clearly stated rationale. Providing schools with high speed access to the Internet solely provides high speed access to the Internet for schools. This, in itself, does not enhance the student's education experience or improve learning outcomes. 


\section{Computers}

Include the number and type of school-owned computers for each location in your school. Please include laptop (L) and desktop (D) computers, as well as thin-client (TC) units, in your count. Use numbers, not words such as all or none.

A multimedia computer is one that has, or is connected directly or by network to, a CDROM drive and can take advantage of a udio and video files stored there.

\begin{tabular}{|c|c|c|c|c|c|c|c|c|c|c|c|c|}
\hline \multirow[b]{2}{*}{$\begin{array}{l}\text { With Intemet } \\
\text { Comnections }\end{array}$} & \multicolumn{3}{|c|}{ In Classrooms } & \multicolumn{3}{|c|}{ In Computer Labs } & \multicolumn{3}{|c|}{$\begin{array}{l}\text { In Shared or } \\
\text { Common Space } \\
\text { (e.g., libraryl }\end{array}$} & \multicolumn{3}{|c|}{$\begin{array}{c}\text { In } \\
\text { Administrative } \\
\text { Offices }\end{array}$} \\
\hline & $\mathbf{L}$ & D & TC & L & D & TC & $\mathbf{L}$ & D & TC & $\mathbf{L}$ & D & TC \\
\hline $\begin{array}{l}\text { Multimed is } \\
\text { Computers }\end{array}$ & & & & & & & & & & & & \\
\hline $\begin{array}{l}\text { Allother } \\
\text { Computers }\end{array}$ & & & & & & & & & & & & \\
\hline $\begin{array}{l}\text { Without Internet } \\
\text { Comnections }\end{array}$ & L & D & TC & L & D & TC & $\mathbf{L}$ & D & TC & $\mathbf{L}$ & D & TC \\
\hline $\begin{array}{l}\text { Multimedis } \\
\text { Computers } \\
\text { With Intemet } \\
\text { Capabilitiss }\end{array}$ & & & & & & & & & & & & \\
\hline $\begin{array}{l}\text { Multimed is } \\
\text { Computers } \\
\text { Without Intenet } \\
\text { Capabilities }\end{array}$ & & & & & & & & & & & & \\
\hline $\begin{array}{l}\text { Allother } \\
\text { Coxnputers }\end{array}$ & & & & & & & & & & & & \\
\hline
\end{tabular}

Table 1: Sample Equipment Matrix (California Department of Education 2001, 62)

\section{The Purpose of Matrices}

The equipment matrix, as a representational method, is part of the planning process for information technology in schools. The matrix is both a powerful and dangerous representation as it can apparently be interpreted in its own right, in a manner disconnected from the body of research and knowledge of which it forms a part. Within the context of a larger text, the matrix also assumes a particular emphasis with its generally graphical presentation. The combination of power and danger that a matrix holds is, also, a consequence of the relationship that connects the matrix with the actual experience of the stakeholders that it purports to represent. Matrices are the extrapolations of particular sets of data that have, in turn, been drawn from a wider accumulation of information and experience (Curriculum Materials Information Services 1997). In the context of information technology, equipment matrices represent certain 'key' features of that experience. This is a focus upon the observable and material features of the equipment in question and particularly 'hardware' and 'software'. This combination of parameters within equipment matrices reflects the power of this form of representation (Tilley, 1989). It combines the simplicity of a data-grid, such as the Cartesian plane, with an apparently meaningful connection of associated and tangible parameters of experience. However, this is an artificial disentangling of what is generally experienced and purchased as a single, albeit somewhat complex, 'thing'.

Utilising equipment matrices in relation to the development of policy concerning information technology in schools requires a minimisation of the dangers found in these representations and a maximisation of their power to present information as coherent snapshots of current experience. These dual requirements present contradictory conditions upon the matrix. The representational power of the matrix decreases as it accommodates an increasing range of parameters. This, in itself, is arguably a commendable means to diminish the political power of the tangible aspects of technology in favour of the less readily represented intangible influences and needs of the stakeholders themselves. A more expansive matrix that represents the intersections of numerous influencing pa- 
rameters approaches an increasingly comprehensive picture of the experience in the classroom and school. As Tufte $(1990,37)$ claims in relation to graphic design, "to clarify, add detail". The ultimate aim for an equipment matrix in the context of schools' IT policy is to enable a model which facilitates rapid self-assessment of a school's current situation and provides a representation that offers means for comparison at inter-school, regional, state and national levels. An equipment matrix developed within this environment will inevitably still remain a representation of actuality although this does not necessarily invalidate its purpose. Even with the necessity to compromise and be characteristic, the equipment matrix requires a range of parameters that extends it beyond 'merely' technical specification in order to model the relationships of technology to human actions and knowledges for the ultimate purpose of policy development.

\section{Two Dimensional Matrices}

A matrix of two dimensions is a particularly restrictive representation of information. As an extrapolation of the variety of human experience with information technology, various classes of information are discarded. Information is disregarded from the individual cases that inform the matrix. This 'simplifies' the matrix by removing variations within each point of analysis that are considered less significant. Within such this narrowed and standardised framework the selection of parameters for each axes in the matrix becomes significant. The seemingly 'obvious' choice for equipment matrix axes is to plot 'hardware' against 'software'. The data that drives these two axes can be quickly defined and collated with little need for detailed consultation with stakeholders. The relationships found at the intersections of the axes of a matrix can later be identified in the machines installed in an education environment after the practical application of policy. The relative experiences of users gathered to inform the design of the system, however, can only be inferred in this form of equipment matrix as it is ultimately 'discarded' in the generalisations of planning. Concern for the intersections of users with technology is subsumed to become a tangent of the relationship between two inter-related parts of what is effectively the same material artefact.

The plot of 'hardware' to 'software' represents a double failure to adequately represent the situation 'on the ground' - in the classroom - beyond enumeration. The 'software' axis does not present a continuum of coherent or sequential data. Where software is first incorporated into a taxonomy that is then introduced en masse into the matrix it is inevitably based upon the intended purpose claimed by its publisher or simplistically categorised by broad computer-oriented tasks, e.g. database, word-processing or spreadsheet. This approach, however, only partially identifies the way software is utilised and understood by learners or their teachers. Production-driven taxa do not present, for example, the possibilities that are available when many separate software packages are used on a single machine. Irrespective of these possibilities, the consideration of software in relation to its capabilities and advantages is generally not predicated on the presence of other software in the system. The primary exception to this situation is found with the advent of the 'Office' suites of software offered by vendors. The advantages of this integration must, however, be offset by the manner in which these suites have traditionally been developed. The majority of vendors offering these suites did not create them 'from the ground up' as integrated packages. The suite is more appropriately assessed as a marketing tool in which a vendor bundles together a collection of software packages that have initially been developed independently of one another in order to claim that it represents the entirety of a user's software requirements.

By incorporating software as a defining axis of an equipment matrix the ability to represent the relationships $b e$ tween software is effectively lost. This loss, at a minimal level, prohibits understanding of the range of software on a specific machine that may, in turn, indicate a general-purpose usage or a specific 'lab' or task context. More importantly, separating software as a distinct unit of specific functions prevents consideration of the interactions that are possible with a mix of software installed on the same machine through mutually readable files 
and 'clipboard' transfers. It is the suite of software found on a particular machine that renders a machine useful or otherwise for a user's particular purpose. A small example of the dangers that the narrow classification of software can produce is found with Microsoft's Word application. While the increasing size of the application and the introduction of arcane features with each new version is a source of criticism for some commentators it does offer some advantages to the student and their teachers. The desktop publishing features of newer Word versions present opportunities for creativity and extension activities in a software environment that is increasingly familiar to many students. However, Microsoft's own classification of this software may actually impede students and their teachers from realising this potential.

Importantly, the significance of particular software and its impact on education can also be demonstrated with Microsoft Word. It is increasingly common for words that have traditionally had alternative spellings in English to be seen as either exclusively US or UK English. This is true, for example, of the ' $\mathrm{s}$ ' or ' $\mathrm{z}$ ' alternative. This can be traced, at least in part, to the spell-checker in Word that determines the ' $z$ ' alternatives are US English and 's' variations to be UK English. However, dictionaries with longer heritages and greater veracity offer a more liberal interpretation without nationalistic parochialism. While this example appears to be a superficial and minor observation it is indicative of the general assumptions made regarding the cultural neutrality of information technology.

Spelling and grammatical differences reflect a wider set of cultural beliefs and understandings that are important to those who use those dialects. Fandrych (2001) has systematically studied the responses to differing language styles made by word processors. The paper concludes, in part, "many first language users even switch off the grammar and spell check options of their word-processors because they do not want to be bothered by the numerous less helpful - and frequently even wrong - suggestions and/or corrections made by the programmers." Longmire (1998) acknowledges the significance of these small observations in a course called, "Language and Cultural Differences: British and American English.” In seeking to understand these differences she asks her students to realise the wider importance of language use and what it reveals about two ostensibly similar cultures.

A 'hardware' axis, in contrast, acknowledges a particular historical and economic relationship between machines. However, this relationship is, again, largely external to the technical requirements of an individual user. The relatively low level of relationship between each point along the axes in a software/hardware equipment matrix maximises the lack of meaning found at the intersections on the matrix. The separation of hardware from software at a policy level of what is treated in the classroom as a single machine, also, creates an artificial representation of meaning that is only abstractly understood and is remote to the classroom environment. Richardson (1989, 172-173) uses Mead's concept of the collapsed act to position and understand artefacts in relationship to its surrounding cultural milieu. The object is not simply 'there' but an integral part of our cultural experience. Richardson $(1989,174)$ claims that, "The stone defines the hand, as surely as the hand defines itself." Richardson presses a claim for the meaningfulness and meaning-ladedness of artefacts. These issues are equally applicable in terms of the hardware of information technology and stresses that these additions to the classroom are as thoroughly enculturated as dictionaries, workbooks, lesson plans and the students themselves. Barns (1991, 897) similarly claims that, "When we talk about 'technologies' it is insufficient to talk only about the manner in which a computer becomes integrated into the practices and discourses of students and lectures. Such artefacts are products of much larger and complex systems involving processes of production, distribution, coordination and so on, which are sedimented within the artefacts themselves."

The hardware/software equipment matrix is not the only two-dimensional representation of a classroom's IT needs - many others are possible. Each combination of factors and the manner of their representation highlights a particular aspect of the classroom experience with information technology. The limitation of these representations, that have been discussed here, emphasises the need to connect the expectations of the user in the 
classroom with the technical capabilities of IT. This requires a multi-dimensional representation of the factors that impact upon the use of IT equipment that does not solely catalogue the equipment within the classroom. Equipment represented within a matrix that is sensitive to users' needs in a classroom is connected to other factors on the learning process as meaningful and single 'things'. The equipment represented in the equipment matrix, in this way, becomes only one axis of the overall picture. This representation remains an equipment matrix though, as its purpose is to identify the range of material requirements necessary to support the acquisition of appropriate IT skills by learners within the classroom. These skills are, in turn, acquired in order to improve the individual learning experience in the context of the curriculum not in the context of the technology itself.

\section{The Consequences of Applying a Two-dimensional Matrices to Policy}

Developing indications of the number of tools, such as computers or video cameras, that a school owns does not, in itself, represent more than a prior financial commitment to specific technology. The presence of equipment within a more sophisticated multi-dimensional matrix, however, implies that it is used in the classroom environment and supports the acquisition of knowledge and skills by students. Education benchmarks derived, at least in part, from matrix-based information must accept these implications and project them forward as the basis for advocacy and planning activities. However, in order to achieve optimum learning outcomes that are supported by supportive information technology policy, the limitations of these representational models must also be acknowledged. The readily interpretable matrix does not itself and in isolation present sufficient context on which to base policy decisions. The policy and the matrix are, in many respects, parallel responses to the same information. Each can assume multiple forms and pursue different directions depending upon what data is incorporated and what is discarded. The matrix can, in this respect, form a graphical representation to the advocacy of policy. More actively, the matrix presents a map on which the significant issues within a policy can be charted for later comparison.

The form and content of the matrix itself determines the extent to which these comparisons can be conducted. A matrix that represents specific equipment information may only offer limited opportunities for extended analysis. Historical comparisons, for example, in the same classroom or school environment may provide indications of the shifting levels of financial support allocated to IT within a school. The basis for this comparison is restricted, however, as it is an almost coincidental aspect of the representation and not the original purpose for the development of a matrix. The limits to analysis within a matrix can be further blurred by differing understandings of what the matrix should represent, what it does represent and the manner in which this representation is applied within the classroom.

The two dimensional representation of IT equipment specification within the classroom and school is capable of one significant capacity - defining the limits of activity without a particular tool. For example, the Internet cannot be accessed without modem, computer and telephone line. The equipment matrix beyond this general observation can only indicate which combinations of equipment fulfil this minimum requirement. These combinations within a matrix can only imply that employed staff and students within a classroom environment also understand the purpose of the equipment and possess the skills to operate it in its intended manner. The use and role of the equipment is influenced by other social factors that are not represented through a 'simple' equipment matrix. This wider complexities not presented in a specifications-oriented matrix is evidenced by the problems schools have experienced connecting to the World Wide Web (see Kapiolani Community College, 1997). The emphasis upon possessing the equipment - an emphasis perpetuated by the specifications-oriented matrix necessary to access the Web is only a small aspect of the continual cost, support and training necessary to ensure an ongoing virtual presence. Regional factors also prevent a 'simple' representation of equipment to learn- 
ing outcomes. The cost of long distance telephone connections is an issue for schools in the Highlands of Scotland, rural areas of the United States and central Australia. Concerns such as these may be hidden within an equipment matrix that seeks to indicate the presence or otherwise of a modem and telephone line in a school environment. Funding allocations and policy decisions that utilise general specifications and per student ratios may only accommodate a generalised need and fail to accommodate specific differences. Similarly, schools gain access to the Internet with a variety of telecommunications constraints for example, 'noisy' lines, congested servers and low bandwidth are not so readily represented within an equipment matrix in contrast to the single tabulation of an 'available telephone socket'. However, the need for the clarity of individual specifications within the equipment matrix approaches the same level of significance for the classroom environment as its actual presence. Congested modem banks at an Internet Service Provider's point of presence can negate the meaning of possessing a modem, telephone line and computer in the classroom. Contemporary aprocryphal stories in the United Kingdom suggest that having 'a broadband connection' can mean, in actuality, having a connection to the Internet that is slower than that available with a conventional modem (George 2002, 28). Similarly, lack of capacity on a hard disk drive can rapidly render a sufficiently fast computer with video-editing software useless for the task implied in its specifications.

At least two factors influence the assessment of a particular technology's relevance and utility to the conduct of specific tasks; how the task is achieved technically (speed, efficiency, system load) and how familiar the sequence of actions needed to successfully undertake the task are to the user's own abilities. In this way, the existent skills, knowledges and capabilities of users must, in some manner, be acknowledged within the equipment matrix to the same degree of significance as 'purely' technical factors. Recommendations and policy decisions cannot solely be based on a catalogue of tools that are available to a school as these provide no contextual indication of who, how or where the tools are to be utilised or for what purpose they are present in the school environment.

Two-dimensional matrices that focus upon technical specifications only, provide a baseline indication of a potential capacity to undertake a given activity within the learning environment. This baseline offers little more support to the decision making and policy-making process than the various cliches used by computer salespeople; "The higher the numbers the better" or "Buy the biggest you can afford". Applied in conjunction with the advice of cynical computer users, "It will all be out-of-date in a couple of years anyway" it is clear that the ad hoc and continuous upgrade cycle that the computer industry heavily encourages is not necessarily the best, or judicious, path for most schools. California's Department of Education (1995) specifically identified the development of ad hoc responses to the implementation of information technology in the classroom and sought in its 1994/95 to specifically address this problem by advocating 'across the board' standardisation. The policy's chapter, "Technical Model for School Networks" consequently reduced the problem of allocating infrastructure to the detailed discussion of specific technologies including Token Ring, Gopher and the Mosaic browser. This chapter lacks any discussion of the education theory driving this development or the rationale for the development of this infrastructure, leaving these issues for other chapters and effectively divorcing the specifics of implementation from the motivations for this investment.

The two-dimensional equipment matrix that focuses upon the 'numbers' encourages and perpetuates this cycle, albeit in a slightly more reserved fashion. A more expansive model that combines other factors influencing the usage of IT within schools can avoid some of the 'snake-oil sale' elements of IT marketing to focus upon the relationship IT to classroom practice and the learning experience.

Leach $(1976,41)$ provides a similar perspective, and caution, regarding isolating and focussing on specific parts of a phenomenon. 
...The participants in a ritual are sharing communicative experiences through many different sensory channels simultaneously; they are acting out an ordered sequence of metaphoric events within a territorial space which has itself been ordered to provide a metaphoric context for the play acting. Verbal, muscial, choreographic, and visual-aesthetic 'dimensions' are all likely to form components of the total message. When we take part in such a ritual we pick up all these messages at the same time and condense them into a single experience which we describe as 'attending a wedding', or 'attending a funeral', [or 'attending a lesson'] and so on. But the analyst must take each dimension by itself, one at a time, and it then become impossible to give a really convincing account of how the different superimposed dimensions fit together to produce a single combined message.

While Leach's discussion relates to the academic researcher examining ritual in a traditional sense and is biased towards performative aspects of the experience the caution is still relevant in the context of information technology. The classroom is a complex environment that similarly requires its participants to condense a variety of messages into a single immediate experience. To isolate the information technology of this experience as a set of policy requirements may not only prohibit a convincing account but prevent the construction of a convincing environment - an environment that supports and promotes the education theory that founds the policy statements.

\section{Technological Determinism}

The purpose of the equipment matrix is to assist a school to develop a well thought-out, intelligent and achievable technology usage plan. If the understanding of IT is reduced to an equipment specification, the IT planner will inevitably be forced to adopt a technological determinist position. Technological determinism occurs when there is a distortion of the focus of the decision-making process away from the people using the equipment to the equipment itself (Jones, 1982). This presents the possibility for planning decisions to be made 'from the brochure' rather than 'from the classroom'. This form of management will tend to support the continued acquisition of equipment as a solution rather than regarding the ongoing impact of, and relationship to, technology by those using, or expected to use, the equipment. A continuous drive to 'have' technology without consideration for previous successes or failures within the individual school environment ties the purchase of new equipment to mainstream, commercial developments. These are developments that are primarily driven by consumer demands to be entertained and to automate or simplify their business requirements. The increasingly patronising interface of Windows XP with no hard edges for users to hurt themselves upon is perhaps a trivial example. The phenomenon is more clearly evidenced in the approach and style of CD-ROM based encyclopedias that have increasingly adopted the Encarta multi-media heavy and information light approach. It is no coincidence that Encarta is Microsoft's product and competes with more traditional, more authoritative and better-regarded encyclopedias. The development of IT capacity from this wider perspective is not oriented towards the facilitation of learning and improvement in the education environment but rather is a reflection of a different series of more commercial demands. The issues and choices related to these potentially competing agendas is discussed specifically in relation to the music used on the Encarta Encycolpedia by Neuenfeldt (n.d.). Neuenfeldt uses examples of the music used to represent specific cultures on Encarta. Particularly revealing are the choices made for English speaking 'Western' cultures such as the use of indigenous music for Australia or the small number of selections used to represent the complex multi-ethnic composition of Belize. In these cases the ubiquitous 'Marketing Department' of Microsoft is impugned as the final arbiter of cultural representations in this encyclopedia. Choices based around commercial value also reflect a desire to be technically up-to-the-minute over the facilitation of a lifelong relationship with information technology tools or lifelong learning in general. The disastrous consequences of this form of planning are well documented through 1970's literature associated with the integration of computers into society (e.g. Jones 1982, Coates 1974). 


\section{Stage-based Methodology - a Hope for Equipment Matrices?}

The Planning for Technology in an International School Report (1998) was developed in the United States as a guideline for international schools and overseas American schools to adopt a technology use plan. The report's advantage is that it was not driven by technology. This rationale was developed, in part, as a response to the limited or 'unknown' resourcing available to this type of school. This need required an intelligent response in the face of minimal resources that emphasises the human aspects of IT. The report advocates determining the current levels of IT use with a resource survey. This survey is expanded to incorporate description of the environment, identification of innovative and the current forms of classroom intervention to launch a school towards the planning process for IT use and integration. The policy that is developed with this process therefore includes a clear rationale, strategy and distinct stages for implementation.

The school environment is not immediately transformed with this type of policy to a 'wired' campus but rather progresses through four stages of technology use. These stages are described by the Planning for Technology in an International School Report (1998) as the "The Emergent Systems Stage", "The Islands of Technology Stage", "The Integrated Systems Stage" and "The Intelligent Systems Stage". This staging of IT usage acknowledges that the policy is not just implementing a change of equipment but a cultural one in which the learning and work environment is being significantly and irrevocably altered. Progression through the four stages in this implementation of IT usage is not simply represented by the accumulation of additional computers. Each stage requires the establishment of formal support systems, plans and procedures. Other considerations that relate to the 'core' business of the school are also considered at each stage. Instructional delivery strategies, budgetary constraints and teachers' relative knowledge are also gauged within the systematic and ongoing adoption of new technologies. Each stage is purposely holistic and aims to incorporate available information technology into the educational environment and culture. This approach prevents, or at least limits, the disruptive potential of new technology in the classroom.

The importance of this stage model is its flexibility. It is self-passed and has the capacity to accommodate the variety of school situations that exist within most education systems. Assessment and policy decisions based on a two-dimensional and specifications oriented representation of a school's situation could not support or inform this integrated planning model.

\section{A Model for Using an Equipment Matrix}

Some general cautions emerge in the use of equipment matrices within IT policies. Any matrix is a representation of available information and not the direct presentation of lived experience in the classroom or elsewhere. With this distance the matrix has the opportunity to assume a variety of forms, each of which emphasises particular concerns and interests. The most powerful, and persuasive, matrices graphically represent physical infrastructural parameters on a Cartesian plane. The issues surrounding IT use in schools, however, constitutes a complex multi-dimensional situation that should not be reduced to this degree.

Within the matrix there is also a need to reflect learners' and teachers' experiences. In the case of IT equipment, the meaningful level of understanding is not found with individual software packages or the peripherals attached to a particular machine. The equipment should be represented within the matrix as it is experienced in the classroom environment, as a single coherent 'thing'. However, the incorporation of equipment specifications directly into the matrix is also a source of difficulty and similarly requires caution. Matrices constructed around technical specifications locate a specific historical moment, which in the case of IT equipment can be measured in weeks or months and certainly in briefer timeframes than those in which policy is developed. In this sense an equipment matrix must provide a longer-term and more appropriate picture of the contemporary computing by indicating 
the general taxa of available equipment. The development of a series of classifications, which could be updated outside the scope of the main matrix, ensures timeliness during the period of the policy's implementation. This would provide flexibility that allows qualitative indications to be represented within the matrix. For example, while two machines with modems both positively correlate to Internet accessibility the relative speeds of this access would require sub-taxa.

The presentation of equipment as taxa within the equipment matrix requires a series of additional parameters against which a relationship can be discerned. The consideration of IT in schools, in an effort to connect educational expectations to technology could incorporate benchmarks of knowledge and skills acquisition. Achievement by a student to a particular benchmark embeds an indication of the specific variety of knowledges and skills they have attained. While these may echo the generic categorisations of software publishing houses, for example word processing and databases skills, it implies a broader social context for these developments. A continuum of benchmarks also indicates 'milestones' when additional IT equipment requirements may be required for the students' continued acquisition of generic skills.

The relationship of IT skills to the equipment that facilitates specific activities that encourage the development of these specific skills incorporates a range of environmental issues. These include factors that generally shape the environment of IT skills acquisition. Among the contributing factors are relative scales of financial and institutional support, aspects of the classroom environment, such as class sizes, and the 'people' aspect of the environment, such as the home ownership of PCs. These considerations can be assessed at a variety of levels from the classroom to a national level.

Considered in context and with an awareness of the learners affected, the equipment matrix ultimately can provide a graphical representation for self-evaluation. The matrix allows for the rapid identification of a variety of inter-relationships, beyond solely technical ones. The acquisition of knowledge and skills provides the only temporal ordering that allows imminent and future requirements to be sensitively identified.

\section{Conclusion - A policy alternative?}

The equipment matrix is a powerful representational tool with the capabilities to persuade. This paper has presented a discussion about the power that infrastructure issues assume within policy documents. The critical focus of this research challenges this emphasis and the tendency to isolate equipment requirements from the theoretical foundations, practical considerations and cultural conditions of school IT policy.

We argue that the heritage of two-dimensional matrices in planning documents has long term impact on contemporary policy and planning. Educationalists and policy makers such as those in California have paid attention to the issues of information technology in the classroom over a relatively long period of time. In responding to changes this state has produced a series of educationally sound and reasoned policy positions through much of the 1990s and to the present day. However, these documents often contain a disjuncture between the advocated educational theory and the means to physically implement these aims in the classroom. In 1995, in Connect, Compute and Compete (California Department of Education), the first telecommunications recommendation was to increase and equip classrooms and libraries in order to improve student achievement. The specific recommendation, however, lost this focus on improving education outcomes and reduced the policy's aim to filling these learning environments with the latest information technology. Generalised policy recommendations that advocate hardware and software purchasing based on averaged projections can impact on the individual outcomes for students and schools. Recommendations and policy decisions cannot solely be based on a "shopper's" catalogue of tools that are currently available to a school. 
This paper has illustrated why equipment matrixes use should be heavily contextualised. In the rush to connect to the World Wide Web, wider social and technical complexities will not be presented in a specifications-oriented matrix. These are, however, complexities experienced by schools regardless of their equipment and infrastructure (see Kapiolani Community College, 1997). The purpose and limits of a matrix and technical specifications in general should be clearly defined. If matrices are not positioned and contextualised carefully they can too readily - due to their simplified and graphical nature - be regarded as a summation and solution to the 'whole' situation.

Equipment matrices and discussions of infrastructure should not be permitted to dominate the debate regarding IT in the classroom. Infrastructure discussions and planning need to continuously return to the purpose and role of a specific technology to assess its contribute to the facilitation of positive learning outcomes. In this respect, the theoretical discussions of Barns (1991) and Richardson (1989) offer some possibilities for more sensitive approaches to the provision of technology infrastructure in schools. If information technology is considered as a series of 'collapsed acts' their introduction into the classroom may be more readily contextualised and appropriately positioned. The introduction of these 'collapsed acts' should be assessed and considered in the same manner as other aids within the classroom environment. The same consideration given to the collapsed acts of a workbook or specific story should also be given to information technology. Just as a text or workbook requires explanation by the teacher to their students so too does information technology. Information technology policy making can learn and gain from the comparative examination of policies and management of other cultural items, or 'collapsed acts' in the classroom. Examples of comparable 'cultural policies' include library acquisition, playground equipment, music lessons and, in the UK and Australia, religious education.

Barns' (1991) communitarian position also offers some direction to policy making in this area. The constant reference point within this philosophical position is to assess and seek the benefit that any given item of technology brings to the community. "For the democratic and communitarian possibilities of new technologies to be realized there will need to be a recovery of those social practices and communal discourses which enable us to flourish as persons and communities and which can effectively 'frame' ongoing technological change" (Barns 1991, 910).

\section{References}

Arnott, Sarah. (2002). Mixed reaction to Blair's promises. Computing (UK), $25^{\text {th }}$ Nov, p.4.

Barns, I. (1991). Post-Fordist People? Cultural meanings of new technoeconomic systems . Futures, November, pp.895-913.

California Department of Education. (1995). K-12 NetworkTechnology Planning Guide, Sacramento, CA: California Department of Education, http://www.cde.ca.gov/edtech/ntpg/

California Department of Education. (1995). Connect, Compute and Compete: The Report of the California Education Technology Task Force, Sacramento, CA: California Department of Education, http://165.74.253.64/edtech/ccc/

California Department of Education. (2001). Education Technology Planning: A Guide for School Districts, Sacramento, CA: California Department of Education, http://www.cde.ca.gov/ctl/edtechplan.pdf

Coates V.T. (1974). Future Directions for Technology Assessment. in Ritterbush P. C. (ed.) Technology as Institutionally Related to Human Values, Washington DC, Acropolis Book

Curriculum Materials Information Services. (1997). Technology and Teaching and Learning - Matrix, Western Australian Department of Education http://www.eddept.wa.edu.au/centoff/cmis/rf413.htm

Curriculum Materials Information Services. (1997). Technology in the Classroom. Western Australian Department of Education, http://www.eddept.wa.edu.au/centoff/cmis/rf411.htm

Fandrych, I. (2001). Word Processors' Grammar and Spelling Assistance: Consequences for Second Language Learning and Teaching. The Internet TESL Journal, Vol. VII, No. 6, June, http://itesli.org/Articles/Fandrych-WordPro.html 
George, Mark. (2002). ADSL woes add up to costly mistake (Letters). Computing (UK), 25 ${ }^{\text {th }}$ Nov, p.28.

Jones, Barry. (1982). Sleepers, Wake! Melbourne: Oxford University Press.

Kapiolani Community College. (1997). Distance Education on the Web: Technology Use Survey, http://library.kcc.hawaii.edu/staff/illdoc/DE/posteval.htm

Leach, Edmund. (1976). Culture and Communication: The Logic by which Symbols are Connected. Cambridge: Cambridge University Press.

Longmire, J. (1998). Language and Cultural Differences: British and American English, Illinois Geographic Alliance Summer Geography Institute, http://www.coe.ilstu.edu/iga/JLlang.htm

Neuenfeldt, K. (n.d.). The Sounds of Microsoft: The cultural production of music on CD-ROMS, Macquarie University, http://www.ccms.mq.edu.au/mus302/readins/sounds of microsoft.html

Planning for Technology in an International School Report. (1998). Stages of Development., John Erhart, Mariposa Resources, http://www.yosemite.net/ jerhart/stages.html

Planning for Technology in an International School Report. (1998), John Erhart, Mariposa Resources, http://www.yosemite.net/ jerhart/modplan.html

Richardson, Miles. (1989). The Artifact as Abbreviated Act: A Social Interpretation of Material Culture. In Ian Hodder (ed). The Meaning of Things: Material Culture and Symbolic Expression. London: Harper Collins Academic.

Tilley, Christopher. (1989). Interpreting Material Culture. In Ian Hodder (ed). The Meaning of Things: Material Culture and Symbolic Expression. London: Harper Collins Academic.

Tufte, Edward. (1990). Envisioning Information, Cheshire CN: Graphics Press. 